\title{
»Engagement ist Mangel an Talent. «1 Zur Entkernung der Kritik in der Kritischen Systemtheorie und dem Postfundamentalismus
}

Niklas Luhmann äußerte einmal, wenn er Jürgen Habermas provozieren könne, dann mit »moralischer Unterkühlung «. Diese Worte sagen einiges aus über die Systemtheorie, über die von ihr vermuteten Gegnerinnen ${ }^{2}$ und nicht zuletzt auch über Luhmann selbst. Die Systemtheorie wurde bereits in der ersten, einschneidenden Debatte mit Jürgen Habermas - aus der diese Bemerkung stammt - zur »Hochform eines technokratischen Bewusstseins « stilisiert. ${ }^{3}$ Ihre wichtigste Gegnerin war denn auch für lange Zeit die Frankfurter Schule, die wiederum bei Luhmann als eine naive, unterkomplexe Wunschliste für eine bessere Gesellschaft angegriffen wurde.

Seit einiger Zeit hat diese klare Gegenüberstellung an Überzeugungskraft verloren. Luhmann selbst formulierte mit Giuseppe Tomasi di Lampedusa, man müsse unendlich viel ändern, damit alles beim Alten bliebe. Er gab sich Mühe, auf Abstand zur Kritischen Theorie zu gehen, der Begriff der »Kritik « stand insgesamt bei ihm für bloßes Wunschdenken. Aber Luhmann ist tot. Auch die Systemtheorie gehört nicht ihrem Schöpfer. Die alte Entgegensetzung zwischen Kritik (Kritische Theorie) und Affirmation (Systemtheorie) weicht der Frage, welche Rolle Kritik eigentlich gesellschaftlich spielen kann und soll. Es zeigt sich ein Wille, beides zu machen, Kritik und Systemtheorie. Dieser findet jüngst programmatischen Niederschlag im Sammelband Kritische Systemtheorie. Zur Evolution einer normativen Theorie, herausgegeben von Marc Amstutz und Andreas Fischer-Lescano. Er bündelt die jüngeren Bemühungen um eine dezidiert emanzipatorische Systemtheorie. ${ }^{4}$ Die Herausgeber weisen den Weg für dieses Unterfangen, wenn sie schreiben:

"Ziel ist die gesellschaftliche Emanzipation, die Demystifizierung von eingerichteten und ausgeübten Hegemoniebetrieben in allen Ecken der polykontexturalen Gesellschaft - und dies mit dem Anspruch, die soziale Responsivität gesellschaftlicher Institutionen herauszubilden. Gegen Tendenzen der Verselbstständigung gesellschaftlicher Institutionen spielt die Kritische Systemtheorie ihre Passion für Subversion, Abweichung und Variabilität aus. Sie setzt im inneren Arkanum der Gesellschaft an, um sechter gesellschaftlich demokratische Selbstreferenz zu gewährleisten und ‘falschen Rechtfertigungszauber zu entlarven. ${ }^{5}$

»Kritisch « meint hier also ein Denken und Theoretisieren, das ausdrücklich auf Emanzipation und Demokratie abzielt. Wenn man dieses Verständnis zweier Theo-

1 Adorno 2003, S. $372 \mathrm{f}$.

2 Aus Gründen der besseren Lesbarkeit verwende ich nur die weibliche Form. Männer sind dabei stets mitgemeint.

3 Vgl. Habermas, Luhmann 1971, S. 145.

4 Im Folgenden: Amstutz, Fischer-Lescano 2013.

5 Ebd., S. 10. 
riekomplexe untersucht, lässt sich in diesem Versuch der Politisierung der Systemtheorie eine allgemeinere Tendenz in der aktuellen Sozialtheorie ablesen: Nunmehr dienen nicht mehr Begriffe wie »Gerechtigkeit «, »Exklusion « oder »Herrschaft « als archimedischer Punkt der Sozialkritik, sondern allein: Kontingenz. Dass das Soziale kontingent sei - also immer auch eine andere Ordnung vorstellbar und die Geschichte kein deterministischer Gang -, wird zum archimedischen Punkt der Kritik.

\section{Die Notwendigkeit der Kontingenz}

»Während Niklas Luhmann es sich in der klimatisierten VIP-Lounge der 27. Beobachterebene mit einem Glas Champagner bequem machte und die emanzipatorischen Kämpfe geschundener Individuen vernachlässigte, stellt kritische Systemtheorie dies vom Kopf auf die Füße. « ${ }^{6}$ Wie genau das bewerkstelligt werden soll, werde ich im Folgenden zeigen.

Entscheidend ist zunächst, dass in obigem Zitat von Fischer-Lescano weniger die Theorie Luhmanns angesprochen ist als Luhmann selbst. Das bietet den Vorteil, die Gründe für die Jahrzehnte der quasi hegemonialen Luhmann-Verortungen (konservativ, sozialtechnologisch, kalt) in der Haltung Luhmanns selbst ausmachen zu können. Luhmann selbst war es, der die Systemtheorie als Gegenpol zur Kritischen Theorie verstand. Luhmann war der Technokrat, Luhmann war der »Dandy « (Fischer-Lescano). Die Kälte und das Dandytum erscheinen offensichtlich als Schwächen, und diese werden an der Haltung des Autors Luhmann festgemacht. Aber die dagegen positionierte kritische Systemtheorie muss gleichsam angeben können, an welchem Punkt sie ansetzen kann. Oder vielmehr: wie jene Akzente gesetzt werden können, die aus der Systemtheorie Kritische Systemtheorie werden lassen.

Will man die vielgestaltigen Vorschläge im Band Kritische Systemtheorie ordnen, so verbindet sie die Einschätzung, dass die Systemtheorie deswegen zur emanzipatorischen, demokratisierenden Kritik in der Lage sei, weil sie »Theorie der Kontingenz « sei. Hierin, in der mit dem Begriff eröffneten Denkbarkeit einer anderen Ordnung und in den Kämpfen, die sich darum entzünden, beweise sich der kritische Geist. Was kontingent ist, kann auch anders sein. Was kontingent ist, ist nicht not-

6 Ebd., S. 15. 
wendig so, wie es ist. ${ }^{7}$ Der Begriff der Kontingenz geht damit in zwei Richtungen auf Distanz: einerseits zur Kausalität, andererseits zum Zufall. Kontingenz unterläuft den Begriff der Kausalität, indem Gesellschaft nicht mehr teleologisch oder kausal vorgestellt wird; die " bestehenden Verhältnisse « sind aus Sicht einer orthodoxen marxistischen Theorie beispielsweise ökonomisch determinierte Verhältnisse. Die ökonomischen Prozesse und Bewegungsgesetze des Kapitals formen die soziale Ordnung, Überbauphänomene wie das Recht, die Politik und die Religion sind lediglich Ausfluss der determinierenden Basis, der Ökonomie. In dieser Hinsicht stellt Marx Hegel vom Kopf auf die Füße: Er lässt das Sein das Bewusstsein bestimmen. Er entwirft eine eigene große Erzählung über Hegel. Von beiden Weisen des Determinismus, von idealistischer wie materialistischer Geschichtsphilosophie, trennt sich der Kontingenzbegriff.

Gleichsam muss Kontingenz mehr meinen als bloße Arbitraritäten, wenn das Nachdenken über sie gleichsam politische Praxis anleiten will. Diese Kontingenz hat nichts zu tun mit dem pejorativen Zerrbild von der Postmoderne, in der letztlich "anything goes « herrscht. Es ist für Luhmann offensichtlich, dass getroffene Entscheidungen und bestehende Strukturen weitere Schritte - in die eingeschlagene Richtung - wahrscheinlicher machen. Luhmann geht freilich nicht antisoziologisch von einer vollständigen Offenheit sozialer Verhältnisse aus. Manches ist wahrscheinlicher als anderes. Es ist kein Zufall, dass ich in diesem Moment dazu komme, diesen Satz zu schreiben. Und doch wäre es auch möglich, ihn nicht zu schreiben. Dies hat Auswirkungen auf die Idee sozialer Transformation: Diese muss vorgestellt werden als bedingte, stückweise Veränderung. ${ }^{8}$

In der Systemtheorie Luhmanns ist die Herrschaft der Kontingenz differenzierungstheoretisch ausgearbeitet. Verschiedene Funktionssysteme operieren nach je eigenen Logiken. Die Wirtschaft folgt offenkundig einer anderen Logik als die Kunst und als die Politik. Luhmanns Systemtheorie radikalisiert diesen Gedanken im Begriff der "Autopoiesis ", also der Selbstreproduktion eines jeden Funktionssystems. Sie alleine, die Systeme, sind es, die die Grenze zwischen sich und ihrer Umwelt in steten Kommunikationen als Sinngrenzen markieren. ${ }^{9}$ Je eigene Welten stehen nebeneinander, die Welt der Wirtschaft als Einheit aus Wirtschaftssystem und dessen Umwelt neben der Welt der Politik als Einheit aus Politik und Umwelt. In den

7 So die Verwendung Niklas Luhmanns, der sich dabei auf Aristoteles bezieht. Vgl. Luhmann 1984, S. 47 und 83 ff. Peter Vogt zeigt anschaulich, dass diese - im derzeitigen Denken offensichtlich dominante - Interpretation historisch freilich einigen Veränderungen unterworfen war. Mal fungierte der Kontingenzbegriff als ein Abkömmling der Möglichkeitstheorie Aristoteles', mal wurde Kontingenz gerade als Gegenbegriff zur bloßen »Möglichkeit «, » possibile « (im Gegensatz zu »contingens «), verstanden. Vgl. Vogt 2011, S. 44-66. Da Luhmann, Marchart, Mouffe - also die entscheidenden Ideengeber dieses Textes - eine solche Diskussion um die ideengeschichtlichen Verwerfungen vermissen lassen, werde auch ich diese hier nicht leisten. Es wäre aber zukünftig spannend, zu sehen, wie die historisch sehr verschiedenen Vorstellungen von Kontingenz Rückschlüsse für die politische Theorie zulassen.

8 Vgl. Marchart 2010, S. 289 ff.

9 Vgl. Luhmann 1984, S. 60 ff. 
Umwelten findet die Verschränkung statt: Die Wirtschaft ist Umwelt der Politik und umgekehrt. Von einem jeden Standpunkt sieht das zu Betrachtende anders aus. Auch ohne das systemtheoretische Vokabular ist dieser Sachverhalt recht einleuchtend: Ich alleine habe genau diese Erfahrungen in genau dieser Reihenfolge gemacht, deswegen bleibt trotz aller strukturellen Analogien und der Parallelität vieler Erfahrungen mein eigenes Leben letztlich genuin nur meines. Die Singularität des Einzelnen (bei Luhmann hieße das: ein psychisches System) ist nicht vollständig aufzulösen in der totalisierenden Allgemeinheit. ${ }^{10}$ Ich kann einzig Erklärungen anbieten, meine Ideen- und Empfindungshaushalte zu übersetzen suchen. Aber Ungleiches lässt sich vergleichen. Die Luhmann'sche Idee einer funktionalen Analyse setzt in der strukturellen Analogie an, die soziale Phänomene daraufhin befragt, welche Funktion sie gesellschaftlich erfüllen. ${ }^{11}$ Ein jedes System wird als Problemlösung beschrieben. Dann kann nach dem jeweiligen Problem gefragt werden. Aber gleichsam bleibt Raum für Kreativität, weil ein jedes Problem auf unterschiedliche Weisen bearbeitet und gelöst werden kann.

"Wie bei jeder Methodenwahl, ja bei jeder Epistemologie, gibt es deutliche Affinitäten zu bestimmten Begriffsdispositionen der Theorie. Hier zielt die Affinität auf Erkenntnisinteressen, die mit Begriffen wie Komplexität, Kontingenz, Selektion angezeigt sind. Die funktionale Analyse benutzt Relationierungen mit dem Ziel, Vorhandenes als kontingent und Verschiedenartiges als vergleichbar zu erfassen. «12

Luhmanns Theorie plausibilisiert anhand des Verhältnisses von einer Beobachterin erster und einer Beobachterin zweiter Ordnung, wie Kontingenz abstrakt fassbar wird. Die Beobachterin zweiter Ordnung beobachtet die Beobachterin erster Ordnung beim Beobachten - und kann dabei sehen, dass die Beobachtung immer blinde Flecken hat. Dies lässt Rückschlüsse zu auch auf das eigene Beobachten: Auch die Beobachterin zweiter Ordnung könnte anders beobachten, eine andere Beobachtung erster Ordnung in den Blick nehmen. Auch die Beobachtung zweiter Ordnung hat einen blinden Fleck. Aufklärung ist damit immer nur als relativer Prozess vorstellbar, Sehen wird immer nur durch gleichzeitiges Nicht-Sehen möglich, Erkenntnis durch eine Beschränkung auf Bestimmtes. Entscheidend ist dabei, dass Identität nur über Differenz möglich ist. ${ }^{13}$ Das bedeutet, dass eine jede Beobachtung von »etwas ", von einem Gegenstand, der offenkundig mit ihm selbst identisch sein soll, nur über die Unterscheidung dieses Gegenstands von anderem möglich ist. Die Beobachterin erster Ordnung beobachtet zwar jeweilig kontingent, indem sie die Unterscheidung je anders treffen könnte, diese Kontingenz allerdings wird erst auf der Ebene der Beobachtung zweiter Ordnung fassbar: "Was Selbstbeschreibungen des Gesellschaftssystems angeht, also des Systems, das in sich selbst Beobachtung erster und Beobachtung zweiter Ordnung ermöglicht, führt der Übergang von der ersten zur

10 Offensichtlich ist dies ein Gedanke in großer Nähe zu Adorno. Zu den Parallelen vgl. Wagner 2005.

11 Luhmann 1984, S. 83 ff.

12 Ebd., S. 83.

13 Ebd., S. $26 \mathrm{f}$. 
zweiten Ebene dazu, die Realität als kontingent, als auch anders möglich zu beschreiben $\ll .{ }^{14}$

Die Beobachtung erster Ordnung kann noch nicht sehen, dass sie zwingenderweise »ignorant « sein muss, weil sie jeweilig nur einen Ausschnitt der Welt sehen kann. Sie muss sich beschränken, sie sieht nicht, was in ihrem Rücken vorgeht. Diese Naivität der Beobachtung erster Ordnung zerfällt, wenn die Beobachterin zweiter Ordnung die Beobachtung selbst beobachtet. Die These, soziale Ordnung beziehungsweise die Gesellschaft sei kontingent, besagt: Ordnung und Historie sind auch anders vorstellbar. Keine unumstößlichen, im Stile von Naturgesetzen sich entwickelnden Kräfte bestimmen die Gesellschaft, zwingen sie in Form. Historisch lassen sich unterschiedliche Deutungen ausmachen, die als determinierende Letztbegründungen fungierten: Gott, der König, das Subjekt, die Rasse markierten in je unterschiedlichen Epochen solche »unbewegten Beweger ", die die vorfindbare Realität auf einen letzten Punkt zurückführen sollten. Für Luhmann ist die Moderne der Eintritt in ein Geschichtsverständnis, in dem Mensch sich der eigenen Ungewissheit gewiss ist/werden kann. Die Moderne ist das Ende der ontologischen Letztbegründungen. Geschichte wird gemacht. Und genau deswegen, weil die Verhältnisse eben auch anders eingerichtet werden könnten, kann gestritten werden. Was kontingent ist, wehrt sich gegen die Abschließung, die Zementierung, die Ontologisierung. In dieser Hinsicht kann dem Kontingenzdenken zumindest ein Widerspruch gegen Biologismus, Determinismus und Fatalismus attestiert werden. Aber ist es mehr? Mit dieser Hoffnung, mit der Hoffnung auf "Emanzipation « zumindest verbindet sich "Kritische Systemtheorie", wie sie Amstutz und Fischer-Lescano skizzieren. Das Ziel ist eine Politisierung, die einerseits die immense Komplexität der Systemtheorie zu nutzen weiß, aber andererseits an der Idee gesellschaftlicher Emanzipation festhält und über die Luhmann'sche Idee einer soziologischen Aufklärung hinausgeht.

\section{Das Politische und seine Kritik}

Zwei Wege sind vorstellbar: Eine jede Kritik, ein jedes normative Urteil muss nicht nur wohlbegründet sein, jede Kritik muss gleichsam über die eigene Standortabhängigkeit Rechenschaft ablegen. Die Kritik muss also selbst erkennen, dass sie kontingent ist. Sie wäre auch anders möglich, sie ist möglich, nicht notwendig genau so, wie sie ist. Das ist Luhmanns Anliegen. ${ }^{15}$ Der andere Weg jedoch, und um diesen geht es nicht nur im Projekt der Kritischen Systemtheorie, sondern auch in dem, was jüngst von Oliver Marchart in zwei einflussreichen Veröffentlichungen »Postfundamentalismus « genannt wurde, deutet Kontingenz als solche als Hebel der Sozialkritik, als dezidiert politische Begrifflichkeit. Wäre dies richtig, wäre Sozialtheorie insofern kritisch, als sie die Kontingenz des Sozialen gegenüber Willkür und Deter-

14 Luhmann 1997, S. 1122.

15 Vgl. Bonacker 2000. Er versucht, einerseits am Begriff der Sozialkritik festzuhalten, andererseits jedoch den Begriff der Kontingenz als Antiessentialismus zu entwickeln, um die Standort- und Zeitabhängigkeit eines jeden (auch kritischen) Urteils zu betonen. 
minismus stark macht. Wie zu zeigen sein wird, droht jedoch eine solche »Metaphysik der Kontingenz « in ihr Gegenteil umzuschlagen: in eine Politik der Entpolitisierung. Und insbesondere Marcharts Einsatz ist kein geringer, seine Verhandlungen in Die politische Differenz versammeln zunächst einige der einflussreichsten politischen Theoretiker der letzten zwei Jahrzehnte unter dem Schlagwort des Postfundamentalismus. 2013 hat er diese Überlegungen mit der Studie Das unmögliche Objekt fortgesetzt, indem er diesen Blick ergänzt um Kapitel von Durkheim über Luhmann und Lyotard bis Adorno. ${ }^{16}$ Marcharts These ist spätestens mit diesem Buch aus 2013 so zu interpretieren, dass das eigene Narrativ vom Postfundamentalismus all diese Ansätze zu integrieren weiß: und der Aufbruch in ein neues Verständnis von Gesellschaft, Politischem und Kritik nur mehr in den Theorien freigeschaufelt werden muss.

In der Figur des Postfundamentalismus wird der Begriff der Kontingenz zu einer Erkenntnistheorie ausgebaut. Die Blüte verschiedener Ansätze entlang der politischen Differenz von Politischem und Politik liest Marchart als Beleg dafür, dass wir gar in eine neue Zeit eingetreten seien, in der sich das Selbstverhältnis grundlegend verändert habe. Deswegen kann er die Moderne in toto auf den Begriff der Kontingenz bringen: »Letztbegründungsphilosophien und -wissenschaften dieser Art (zum Beispiel von der Art des marxistischen Traditionalismus) gelten heute als ausgestorben $" .{ }^{17}$ An diese steile, wenngleich auch vor allem rhetorisch gesetzte These schließt Marchart mit den Worten an: "Doch man darf sich nicht täuschen. Der Fundamentalismus lebt in vielen Ansätzen fort - man denke nur an den Neoliberalismus und dessen Behauptung von den unabänderlichen Naturgesetzen des Marktes oder an all die hochsubventionierten Versuche, soziale und psychologische Identitätsmerkmale in den menschlichen Genen auszuspüren «. ${ }^{18}$

Marchart bringt die »Notwendigkeit der Kontingenz « des Sozialen auf die Formel "das Politische «. Dieses ist keine empirisch-historische Kategorie, sondern die Bedingung der Möglichkeit »der Politik «. Das Politische gibt der konkreten, empirischen Politik Raum und Sinn, man könnte auch sagen: Es ist ihr Äther. Während es in der Politik um die Verwaltung von Kausalitäten und Sachzwängen geht, geht es im Politischen um die Öffnung, um Möglichkeiten und Denkbarkeiten sozialer Umgestaltung. Die Ontologie des Politischen (also notwendiger Kontingenz) steht der »Ontik « der Politik gegenüber - so eine Unterscheidung Mouffes im Anschluss an Heidegger. ${ }^{19}$ Interessant bei dieser Perspektivierung ist, wie sich erkenntnistheoretische und normative Argumente verschränken: Einerseits markiert der Postfundamentalismus die epistemologische Einsicht, dass Erkenntnisse jeweilig konstruiert werden, dass also die jeweils eingenommene Beobachtungsperspektive auf die Gesellschaft kontingent ist. Der ökonomistische Determinismus des Marxismus ist ebenso wie der naturalistische Determinismus der Soziobiologie mit Luhmann

16 Vgl. im Folgenden Marchart 2010; Marchart 2013.

17 Marchart 2010, S. 15.

18 Ebd.

19 Vgl. Mouffe 2007, S. 15 ff. 
zementierte Beobachtung erster Ordnung - beide Determinismen können nicht sehen, dass sie nicht sehen können. Demgegenüber soll (normativ!) die Theoriebildung selbst ihre eigene Beobachtungsabhängigkeit mitdenken - weswegen auch die Systemtheorie dann bloß einen Beginn neben anderen darstellen kann. Kontingenz wird einerseits zum ohnehin vorherrschenden Prinzip der Reflexion und andererseits zum ethischen Gebot, zum Einfordern eines allgemeingültigen Pluralismus. Die Identität einer sozialen Gruppe, ihr Wertekanon ist nur vorstellbar als Ausschluss von Anderem, von auch Möglichem - Demokratie ist nur zu denken als Grenzziehung gegenüber der Nicht-Demokratie, das System nur im Unterschied zur Umwelt. Gleichzeitig sind die Differenztheorien Luhmanns und Marcharts auch ein Appell für die Toleranz gegenüber der Abweichung und gegen die Idee eines universalistischen "zwanglosen Zwangs des besseren Arguments " (Arendt und Habermas).

Ist also Kontingenz allein dadurch politisch, dass sich in ihr - womöglich - der Wunsch nach Toleranz festmacht? Weil sich Welt auch anders beobachten lässt, weil Du X anders siehst als ich, gilt es, Toleranz zu üben. So wäre zu hoffen. Wenn sich die politische Dimension des Kontingenzbegriffs in der Toleranz gegenüber der Abweichung erschöpfte, bestünde das politische Moment im moralischen Votum für einen erkenntnistheoretisch flankierten Pluralismus. Chantal Mouffe jedoch wendet sich in ihrer Studie Über das Politische, die die Marchart'sche Parallelisierung der politischen Differenz mit der Heidegger'schen ontologischen (Sein und Seiendes) vorwegnimmt, noch dezidiert gegen eine solche Identifikation des Moralischen mit dem Politischen. ${ }^{20}$ Die Unterscheidung politischer Gegner sei eben nicht identisch mit der Distinktion von Gut und Böse. Die Moralisierung politischer Auseinandersetzung bewirke letztlich das genaue Gegenteil, sie laufe auf eine Totalisierung eigener Perspektiven zurück - der Mächtigere diktiere hinter dem Schutzmantel der Forderung nach Toleranz.

\section{Alles ist politisch!}

Kontingenz eröffnet einen Spielraum. Kontingenz verdeutlicht, dass anderes denkbar wäre. Aber mit dem Bewusstsein der Kontingenz geht auch eine intellektuelltheoretische Einsicht einher, die das politische Handeln überhaupt erst möglich macht: Wer beispielsweise glaubte, die Herrschaftsverhältnisse zwischen den Geschlechtern gestalteten sich so, weil die Natur des Menschen sie genau so erzwingt, dem bliebe für die politische Umwälzung allerhöchstens noch der Schritt in die Genetik. Kontingenztheorie kann demgegenüber den Diskurs - beispielsweise jenen um die Natürlichkeit bestimmter Rollen und Kräfteverhältnisse - als solchen zu reflektieren versuchen. ${ }^{21}$ Damit ist aber Kontingenz freilich nicht automatisch schon politisch. Sie mag verweisen auf das Politische, welches dann steht für die Kontingenz sozialer Ordnung. Aber diese Aussage ist trivial. Wer für Pluralismus eintritt, der erkennt die Kontingenz auch eines jeden Urteils (kritisch, konservativ,

20 Vgl. ebd., S. $100 \mathrm{ff}$.

21 Ich denke dabei natürlich zuvorderst an Butler 1991. 
liberal usw.) an. Aber ist umgekehrt jede Kontingenz schon eine Öffnung hin zu einer demokratischen, pluralistischen Gesellschaft? Und ist damit schon ein jeder Hinweis auf die Kontingenz eines gesellschaftlichen Phänomens - des Kleides in Rosa, der Debatte um das bedingungslose Grundeinkommen, der Umstellung von Studiengängen auf Bachelor und Master - eine kritische (emanzipatorische, demokratisierende) Geste?

Nicht jede Kontingenz wirft Fragen zur Gestaltung und Umgestaltung sozialer Ordnung auf, nicht jede Kontingenz wird problematisch, nicht jede Kontingenz ist identisch mit politischer Intervention. Es lässt sich lediglich festhalten, dass eine Demokratie sich als notwendig kontingent denken muss, sie muss also immer offen bleiben für eine andere Ausgestaltung. Bis hierhin ist einzusehen, dass Kontingenz als Denkfigur den Raum eröffnen kann, in dem Politisierung betrieben werden kann, auf dessen Grundlage das faktische, politische Engagement einsetzen kann. Diese Politisierung jedoch muss dann erst noch in der einen oder anderen Richtung geleistet werden.

Die Kontingenz sozialer Ordnung wird immer dann vernehmbar, wenn sich Differenzen, wenn sich Alternativen zeigen, Kritik geübt und gestritten wird darüber, wie wir leben wollen. Einerseits macht Kontingenz den Konflikt möglich, weil es denkbar wird, anders zu ordnen. Andererseits macht der Konflikt Kontingenz sichtbar, in den Situationen der Spannung tritt die Fragilität sozialer Ordnung in den Blick - die Fassaden beginnen zu bröckeln. ${ }^{22}$ Aber gilt dies immer und überall? Ist bereits der Streit über den nicht beschnittenen Apfelbaum ein Hinweis auf das Politische? Marchart schreibt: »Eine politische Ontologie hat Auswirkungen auf unsere Vorstellung noch von den geringsten, bescheidensten und privatesten Handlungen. Auch sie sind Äußerungsform des Politischen [...]«. Weiter: »Deshalb konnte der Foucault der Genealogie zu Recht sagen: salles ist politisch`, weil alles nämlich von Macht- und Kräfteverhältnissen durchdrungen ist «.23

Entscheidend ist die Reichweite, die Marchart der politischen Sphäre begrifflich gönnt: alles ist politisch. Marchart hat Foucault als Gewährsmann. Die Passage, von der Marchart spricht, zeigt Foucault im Interview, in dem er antwortet auf die Frage, was das Politische ausmache. Er antwortet:

"Wenn es stimmt, dass die Gesamtheit der Kraftverhältnisse in einer gegebenen Gesellschaft den Bereich des Politischen konstituiert und dass eine Politik eine mehr oder weniger globale Strategie ist, die diese Kräfteverhältnisse zu koordinieren und zu finalisieren versucht, dann kann man, glaube ich, Ihre Fragen auf folgende Weise beantworten: Die Politik ist nicht das, was elementare und von Natur aus neutrale Beziehungen in letzter Instanz determiniert (oder überdeterminiert). Jedes Kraftverhältnis impliziert zu jedem Zeitpunkt eine Machtbeziehung (die gewissermaßen dessen momentaner Querschnitt ist), und jede Machtbeziehung verweist, insofern das ihre Wirkung, aber auch insofern das ihre Bedingung der Möglichkeit ist, auf ein politisches Feld, dem sie angehört. Sagt man, ‘alles ist politisch', so behauptet man damit diese Allgegenwart der Kraftverhältnisse, und dass sie einem politischen Feld immanent sind; doch erlegt man sich damit die noch kaum umrissene Aufgabe auf, dieses endlose Durcheinander aufzudröseln. « 24

22 Vgl. Marchart 2013, S. $31 \mathrm{ff}$.

23 Ebd., S. 431.

24 Foucault 2003, S. 305. 
Hier kommt der jeweilig politisierende oder durchaus auch umgekehrt entpolitisierende Prozess selbst in den Blick. Auch in der scheinbar wertneutralen Beschreibung sozialer Realität kommt es zu einer notwendigen Verengung, zu einer Reduktion von Komplexität, und hierbei spielt auch die politische Theorie eine entscheidende Rolle, indem sie den Blick lenkt, die Augen öffnet (wofür, das bleibt kontingent) oder sie verschließt. So politisieren und entpolitisieren Theorien. Foucaults Antwort in diesem Interview weist eine beachtenswerte Fähigkeit zur Reflexion dieser notwendigen Verschränkung von einer Theorie des Politischen und einer im wahrsten Sinne des Wortes politischen Theorie auf. Er formuliert bewusst die passive Formulierung: »Wenn es stimmt, dass ... «, und, einschneidender noch: "Sagt man ‘alles ist politisch «, so behauptet man ...«. Foucault markiert den Akt seines Sprechens, er markiert die ihrerseits notwendig kontingente Ontologisierung der Behauptung "alles ist politisch «.

Und tatsächlich sind freilich auch die Momente, in denen Kontingenz vernehmbar, sichtbar, hörbar wird, selten. Die Marchart'sche Adelung auch der »geringsten, bescheidensten und privatesten Handlungen « zu einem genuin politischen Phänomen läuft auf eine »Hyperpolitisierung « (Derrida) des Sozialen zu. Nicht nur wird so der fraglos graduelle Unterschied, der zwischen der Wahl meines Internetbrowsers und einer politischen Partei besteht, verwischt. Erstens ist nicht jede Kontingenz vernehmbar. Zweitens führt nicht eine jede vernommene Kontingenz zu einem politischen Konflikt, in dem um Demokratie und Freiheit gestritten würde. Eine jede Form der Zurückweisung von Zumutungen wird in einem solchen, »totalen « Kontingenzdenken unterschiedslos zu einem Akt der Emanzipation stilisiert.

Während aber manche Denkbarkeit dauerhaft ungedacht bleibt, entfacht eine andere heftigste Diskussionen, soziale Kämpfe gar. Aus der Möglichkeit, über das aktuelle Sein hinauszudenken, wird nur selten eine Frontstellung, in der ein Sollen gegen dieses Sein ins Feld geführt wird. Dies hat auch Auswirkungen auf die Konflikte, die sich an einer jeden Denkbarkeit entspinnen können: ${ }^{25}$ Manchmal, sehr selten kommt es zu überaus intensiven Konflikten, zur Gewalt. Sie erreichen zum einen insofern eine andere Intensität, als sie eine größere Anzahl von Menschen involvieren. Zum anderen ist mit weiteren, heftigeren Konsequenzen zu rechnen, aus Gegnern werden Feinde. ${ }^{26}$ Die harmlose Zurechtweisung (zum Beispiel jener, die nicht in Kontingenzen argumentieren), eine soziale Sanktion weicht echter, physischer Gewalt.

Indem Marchart das Politische totalisiert, muss er einer "minimalen Politik « das Wort reden: Weil alles politisch ist, ist auch das Nichtstun Politik, weil alles politisch ist, ist kindlicher Trotz funktionales Äquivalent zu den Provokationen des Westens durch Nordkorea. Auch der Protest des Kleinkinds gegen das Füttern wird zur widerständigen Praxis, ja gar »Revolte «. ${ }^{27}$ Was auf der epistemologischen Ebene des Politischen begrifflich Sinn macht, ist als politische Intervention, als konkrete

25 Vgl. Marchart 2013, S. 203 ff.

26 Damit reicht das Politische als Konfliktraum mindestens von Mouffes Gegnern bis zu Schmitts Feindschaften.

27 Foucault 2003, S. 525. 
Politik gefährlich. Marcharts Hyperpolitisierung einer jeden Differenz bewirkt dadurch das Gegenteil: die Verkennung aller Intensitäten, die Gleichsetzung verschiedenster sozialer Praktiken. Die soziale Kritik kann dadurch nicht mehr sinnvoll Prioritäten setzen, an wichtigeren gegenüber weniger wichtigen Druckpunkten ansetzen. Marxistisch formuliert: Sie kann die Unterscheidung von Haupt- und Nebenwidersprüchen nicht mehr leisten. Die Folge der Hyperpolitisierung ist ein vager, im Allgemeinen bleibender Wunsch nach Emanzipation.

\section{Theorie machen wie Punk}

Bei allen Bekenntnissen - diese jüngeren Versuche, aus dem Abstrakten, aus der Metaphysik des immer auch anders Denkbaren Funken zu schlagen, bleiben mutlos, sie verweigern sich dem Experiment und freilich auch dem Risiko. Luhmann schrieb mit Bezug auf die ökologischen Bewegungen der 1980er Jahre:

»Gegenüber einer Moral, die angstbezogene Unterscheidungen propagiert, haben theoretische Analysen einen schweren Stand. Angst ist, da sie die Ungewißheit der Sachlage in Gewißheit der Angst transformiert, ein selbstsicheres Prinzip, das keines theoretischen Fundaments bedarf. Sie kann, und zwar mit Recht, die Theorien dem Funktionssystem Wissenschaft zurechnen und sie danach unterscheiden, ob sie mit der Angst sympathisieren oder nicht. Die Beobachtungsposition einer Rhetorik und Moral, die sich auf Angst gründet, hat in der Nachfolge des alten Apriori der Vernunft eine unanfechtbare Selbstsicherheit. « ${ }^{28}$

Die systemtheoretische Ausgliederung des Menschen in die Umwelt sondert mit diesem auch den Affekt aus. Und Luhmann bringt unter der Hand mit der Gegenüberstellung von Theorie einerseits und Angst andererseits dann doch wieder einen Rationalismus (/Philosophismus/Theoretizismus) in Stellung, den viele Systemtheoretikerinnen überwunden zu haben glauben. Luhmann trennt nicht nur Theorie und Affekt voneinander, er identifiziert gleichzeitig die grüne Protestbewegung, die eine andere Gesellschaft einfordert, mit dem Affekt und kann dann die Theorie triumphal auf die andere Seite stellen. Dass sich in der Denkbarkeit, in der Nicht-Notwendigkeit sozialer Verhältnisse nicht automatisch auch ein Denken gegen die faktischen Kräfteverhältnisse ablesen lässt, zeigt sich gerade in der Luhmann'schen Theorie. Über sein eigenes Vorgehen schreibt er: »Die funktionale Analyse benutzt Relationierungen mit dem Ziel, Vorhandenes als kontingent und Verschiedenartiges als vergleichbar zu erfassen. Sie bezieht Gegebenes, seien es Zustände, seien es Ereignisse, auf Problemgesichtspunkte und sucht verständlich und nachvollziehbar zu machen, daß das Problem so oder auch anders gelöst werden kann «.29

Luhmanns Systemtheorie kanzelt mit dem Kontingenzbegriff den sozialen Veränderungswillens geradezu ab: »Der vorherrschende Trend soziologischer Forschung [...] beschränkt sich darauf, unbehagliche Kausalitäten, latente Funktionen usw. einfach bloßzustellen. Man nennt das >kritisch aber nur zu der Frage, wie denn nun die zu Grunde liegenden Probleme anders gelöst

28 Luhmann 2004 [1986], S. 246.

29 Luhmann 1984, S. 83 f. 
werden können «. ${ }^{30}$ Luhmann fordert also ein: »Erst mal besser machen«. Das ist ein immens hoher Anspruch an eine jede Kritik. Man könnte auch sagen: Luhmann überfordert die Sozialkritik bewusst mit einem solchen Anspruch. In der Systemtheorie wird Kontingenz so in Stellung gebracht, als Kritik der Kritik, als Zersetzung aller Utopie. Es würde gleichzeitig immer alles besser und schlechter, so lautet eines der späten, ziemlich ratlosen Statements von Niklas Luhmann in einem Interview. Kontingenz ist offensichtlich zu wenig. Bereits in der quasi-hegemonialen Behauptung der »Notwendigkeit der Kontingenz « schlummert schlussendlich die radikale Vereinfachung des "alles ist politisch «.

Hier bedürfte es des Mutes. Mut, die eigene Position zu markieren, für mehr einzustehen als nur für ein Denken in Kontingenzen. Mut zur empirischen Konkretion und damit auch zur Bereitschaft, bestimmte Kausalitäten, Kräfteverhältnisse, Hierarchien zu benennen. Das war noch das Programm der Kritischen Theorie erster Generation bei Horkheimer und Adorno. ${ }^{31}$ Das Ziel ist eine Theorie, die praktisch wirksam ist, die konkrete Missstände benennt. ${ }^{32}$ Vor allem warnte Adorno (wie auch Walter Benjamin) stets vor der totalen Kontrolle des Individuums, der Einzelnen, des Besonderen durch die verwaltete Welt, das Allgemeine.

Für eine so gestaltete Verschränkung von Theorie und Praxis kann die Systemtheorie Luhmanns ebenso wenig genügen wie die Marchart'sche Identifikation des Politischen mit Kontingenz. Dieses Politische ist ohne politischen Gehalt, es ist leer und unbestimmt. ${ }^{33}$ Der Postfundamentalismus, zu dem sich auch die Kritische Systemtheorie bekennen könnte, versagt sich eine klare Positionierung, er versucht beides, nicht technokratisch-kühl zu sein und nicht simplifizierend, kritisch, aber nicht normativ. Das ist zu wenig, um sich Kritik nennen zu können. Denn Kritik muss sich dem Konkreten widmen, der Bestimmung von Unrecht, der Empörung über bestimmte Kräfteverhältnisse. Emanzipation? Wo und für wen?, muss man fragen. Aber die Abstraktion, für die das Kontingenzdenken programmatisch steht, bleibt distanziert, als ob sie außerhalb stehen könnte. Dann kann aber nur noch gesprochen werden vom »Elend der Welt «, von »dem Menschen « und »dem Leid « oder »dem Kapitalismus «. Die andere Form der Abstraktion, sie wird auch in den jüngeren Diskussionen um die Notwendigkeit der Kritik wieder bemüht, ${ }^{34}$ übt sich in immer neuen Volten, in der "Soziologie der Kritik «, in der » Theorie der Kritik «

30 Ebd., S. 85, Fußnote 109.

31 Es muss hier nicht geklärt werden, ob die »Dialektik der Aufklärung « den Glauben an praktische Wirksamkeit und die Möglichkeit der Veränderung zum Besseren nicht aufgegeben hat.

32 Vgl. Horkheimers programmatischen Text »Traditionelle und kritische Theorie«. Die Pointe dieses Verständnisses ist es gerade, dass die Theorie immer über sich selbst hinausweist. Oder anders: dass sie selbst weiß, dass auch die Theorie eine soziale Praxis ist und damit niemals »neutral «. Theorie, die dies abstreitet, entpolitisiert beziehungsweise ist nach Horkheimer Ideologie.

33 Vgl. auch Wagner 2014, die in ihrem Text genau für die Unbestimmtheit der Kritik normativ - zu votieren scheint.

34 Vgl. überblickend über jüngere Kritikverständnisse beispielsweise Jaeggi, Welsche 2009; Bröckling 2013. 
usw. Positivismus ist wieder Schimpfwort geworden, und doch, Kritische Theorie zeigt sich nach sozialem Konstruktivismus, Beobachtungs- und Differenzierungstheorie (also den drei Säulen der Luhmann'schen Systemtheorie) so geläutert, so auf Unangreifbarkeit bedacht, dass sich der kritische Geist vor allem darin zeigen soll, wie geschickt er alle anderen Kritikverständnisse als »naiv ", » anachronistisch « oder gar »alteuropäisch « (Nietzsche und Luhmann) abtun kann. Hartmut Rosa konstatiert schön in einem aktuellen Interview: »Und die zeitgenössischen Nachfolger [der Kritischen Theorie erster Generation] heute kommen gar nicht mehr zu diesem Kerngeschäft, weil sie nur noch um die Frage der Methode kreisen. Sie klären mühselig, was die Bedingungen der Möglichkeit der Kritik sind «. .35

Womöglich ist die eigentliche Lehre des Postfundamentalismus, dass auch die Kritik ihre eigenen Dekonstruktionen, Relativierungen, Gegenargumente gleich mitzuliefern hat. Genau hierfür steht die Idee, in der Kontingenz ließe sich bereits die Richtung finden, schon hierin liege der archimedische Punkt der Sozialkritik begründet. Schon der Ausweis von Potenzialitäten wird zur kritischen Geste gemacht. Und doch, was Kritik heißen will, muss bekennen, sich ins Getümmel begeben. Beobachtete Luhmann noch aus sicherer Höhe die verloschenen »Vulkane des Marxismus ", so soll auch die Kritik im Zeitalter des Postfundamentalismus lediglich Paradoxien anzeigen, Möglichkeiten eröffnen. Das ist nicht wenig. Aber ein solches Verständnis von Kritik kann sich höchstens kritisch nennen in Bezug auf eine Kritik erster Ordnung, der mit immer wieder besserwissendem (hier: Luhmann'schem) Gestus vorgehalten werden kann: Du kannst nicht sehen, was Du nicht sehen kannst. Eine solche Idee ist eine entkernte, eine formalistische MetaKritik. Sie lässt sich einlullen von der Faszination, dass »die moderne Gesellschaft « eben doch erstaunlich gut funktioniert. Die Komplexität wird zum Eigenwert, zum Fetisch.

Hinter dieser Vorsicht steckt ein Wille zum System, zu den in sich geschlossenen Großtheorien. Trotz des Einflusses der Differenztheorien, die stets "das Andere " gegen Systemwillen und Identitätsdenken ins Feld führen, trotz der Prominenz von Žižek, von Judith Butler, von Alain Badiou dominiert bei Marchart, Laclau, Mouffe schlussendlich doch das geschlossene Weltbild. Jede direkte Einmischung in das Geschehen, in die Praxis stellt dabei ein Risiko dar, jede These könnte in einem späteren Text beziehungsweise im Systemganzen zum Problem werden. So wird die Autorin zur Sklavin der eigenen Schöpfung. Nichts fürchtet die Kritikerin mehr als die Gefahr des Widerspruchs. Die Folge ist mal der Verzicht auf Thesen, mal die Abstraktion. Um Gegenteiliges zu bewerkstelligen, um also eine Kritik - wahrlich - aus der Mitte heraus vorzubringen, bedürfte es des Geistes - sagen wir - Wittgensteins, der sich mit seinem zweiten Hauptwerk Philosophische Untersuchungen radikal gegen seine Theorie der idealen Sprache aus dem ersten Hauptwerk, dem Tractatus logico-philosophicus, wandte. Darin drückt sich nicht nur der Mut zur Differenz und zum Dissens mit der »Community « aus, sondern radikaler noch: der Widerspruch gegenüber den eigenen Worten und Gedanken, manchmal gar die Abkehr vom (alten) System. Wirklich kritisch, ja befreiend wäre es, flexibel zu blei- 
ben, ohne Rücksicht auf Vorheriges, auf die eigene Theoriensozialisation. Wir sollten Theorie machen wie Punk. ${ }^{36}$ Aus einem Gefühl der Aktualität heraus, auf den Punkt, ohne Rücksicht. Kritisch ist es, in diesen einen Text einhundert Prozent Überzeugung hineinzulegen, aktuell, bewegt, direkt. Und dann zu sehen, wohin diese einen trägt.

\section{Literatur}

Adorno, Theodor W. 2003. Ästhetische Theorie. Frankfurt a. M.: Suhrkamp.

Amstutz, Marc; Fischer-Lescano, Andreas 2013. Kritische Systemtheorie. Zur Evolution einer normativen Theorie. Bielefeld: transcript.

Bonacker, Thorsten 2000. Die normative Kraft der Kontingenz. Nichtessentialistische Gesellschaftskritik nach Weber und Adorno. Frankfurt a. M., New York: Campus.

Bröckling, Ulrich 2013. "Der Kopf der Leidenschaft. Soziologie und Kritik «, in Leviathan 41, 2, S. 309-323.

Butler, Judith 1991. Das Unbehagen der Geschlechter. Frankfurt a. M.: Suhrkamp.

Foucault, Michel 2003. Schriften in vier Bänden. Dits et Ecrits III. Frankfurt a. M.: Suhrkamp.

Habermas, Jürgen; Luhmann, Niklas 1971. Theorie der Gesellschaft oder Sozialtechnologie Was leistet die Systemforschung? Frankfurt a. M.: Suhrkamp.

Jaeggi, Rahel; Welsche, Tilo 2009. Was ist Kritik? Frankfurt a. M.: Suhrkamp.

Luhmann, Niklas 1984. Soziale Systeme. Grundriß einer allgemeinen Theorie. Frankfurt a. M.: Suhrkamp.

Luhmann, Niklas 1997. Die Gesellschaft der Gesellschaft. Frankfurt a. M.: Suhrkamp.

Luhmann, Niklas 2004 [1986]. Ökologische Kommunikation. Kann die moderne Gesellschaft sich auf ökologische Gefährdungen einstellen? Wiesbaden: VS Verlag für Sozialwissenschaften.

Marchart, Oliver 2010. Die politische Differenz. Zum Denken des Politischen bei Nancy, Lefort, Badiou, Laclau und Agamben. Berlin: Suhrkamp.

Marchart, Oliver 2013. Das unmögliche Objekt. Eine postfundamentalistische Theorie der Gesellschaft. Berlin: Suhrkamp.

Mouffe, Chantal 2007. Über das Politische. Wider die kosmopolitische Illusion. Frankfurt a. M.: Suhrkamp.

Vogt, Peter 2011. Kontingenz und Zufall. Eine Ideen- und Begriffsgeschichte. Berlin: Akademie Verlag.

Wagner, Elke 2005. "Gesellschaftskritik und soziologische Aufklärung. Konvergenzen und Divergenzen zwischen Adorno und Luhmann «, in Berliner Journal für Soziologie 15, 4, S. 37-54.

Wagner, Elke 2014. »Medialität der Kritik. Die Herausbildung kritischer Sprecher über mediale Unbestimmtheit«, in Leviathan 42, 1, S. 94-114.

36 Diese Formulierung lehnt sich bekanntermaßen an Paul Feyerabends Votum für »Wissenschaft als Kunst« an. 
Zusammenfassung: Dieser Beitrag untersucht, was auf dem Spiel steht, wenn der Satz »alles ist politisch « hegemonial wird. Genau dies passiert, so wird argumentiert, wenn bereits aus dem Hinweis auf die Kontingenz sozialer Ordnung(en), Institutionen, Ideen gefolgert wird, damit verbinde sich bereits eine Kritik beziehungsweise eine emanzipatorische Politisierung. Anhand der Beispiele der Kritischen Systemtheorie und des Postfundamentalismus wird dies plausibilisiert und gegen diese Ansätze für einen stärkeren Kritikbegriff votiert, der die Konkretion anstrebt und so zu Differenzierungen in der Lage ist. Dahinter steht die Auffassung, dass Politisierung immer auch das Setzen von Prioritäten meinen muss.

Stichworte: Kontingenz, Kausalität, Systemtheorie, Politisierung, Theorie und Praxis

\title{
»Commitment is a lack of talent. « The gutting of critique in critical systems theory and postfoundationalism
}

Summary: This article examines the consequences of the statement »everything is political « in theory and practice. The argument is that this happens when references to the contingency of social order(s), institutions and ideas are made and the results are connected to criticism and an emancipatory politicization. By discussing prominent examples of critical systems theory and postfoundationalism, a stronger conception of critique is proposed, which is concerned with empirical concretion. Politicization is therefore only possible by setting priorities.

Keywords: contingency, causality, systems theory, politicization, theory and practice

\author{
Autor \\ Nicklas Baschek \\ Diplom-Politikwissenschaftler \\ Universität Hamburg \\ Institut für Soziologie \\ Privat: Neustadtscontrescarpe 54 \\ 28199 Bremen
}

